\title{
Further studies on the role of phospholipids in determining the characteristics of mitochondrial binding sites for type I hexokinase ${ }^{*}$
}

\author{
Jan Hutny ${ }^{\bowtie}$ and John E. Wilson
}

Department of Biochemistry, Michigan State University, East Lansing, MI 48824-1319, U.S.A.

Received: 17 May, 2000; revised: 10 August, 2000; accepted: 29 August, 2000

Key words: hexokinase, mitochondria, mitochondrial binding, phospholipids

\begin{abstract}
Previous work has indicated that two types (A and B) of binding sites for hexokinase exist, but in different proportions, on brain mitochondria from various species. Hexokinase is readily solubilized from Type A sites by glucose 6-phosphate (Glc-6-P), while hexokinase bound to Type $B$ sites remains bound even in the presence of Glc-6-P. Type A:Type B ratios are approximately 90:10, 60:40, 40:60, and 20:80 for brain mitochondria from rat, rabbit, bovine and human brain, respectively. The present study has indicated that $\mathrm{MgCl}_{2}$-dependent partitioning of mitochondrially bound hexokinase into a hydrophobic (Triton $\mathrm{X}$-114) phase is generally correlated with the proportion of Type B sites. This partitioning behavior is sensitive to phospholipase C, implying that the factor(s) responsible for conferring hydrophobic character is(are) phospholipid(s). Substantial differences were also seen in the resistance of hexokinase, bound to brain mitochondria from various species, to solubilization by Triton X-100, Triton X-114, or digitonin. This resistance increased with proportion of Type B sites. Enrichment of bovine brain mitochondria in acidic phospholipids (phosphatidylserine or phosphatidylinositol), but not phosphatidylcholine or phosphatidylethanolamine, substantially increased solubilization of the enzyme after incubation at $37^{\circ} \mathrm{C}$. Collectively, the results imply that the Type A and Type B sites are located in membrane domains of different lipid composition, the Type A sites being in domains enriched in acidic phospholipids which lead to greater susceptibility to solubilisation by Glc-6-P.
\end{abstract}

The Type I isoenzyme of mammalian ferase, EC 2.7.1.1) is ubiquitously expressed hexokinase (ATP:D-hexose 6-phosphotrans- in mammalian tissues [1] but found at particu-

\footnotetext{
This work was supported by NIH Grant NS 09910.

${ }^{\square}$ Address for correspondence: Jan Hutny, Department of Biochemistry, Agricultural University of Wrocław, K.C. Norwida 31, 50-375 Wrocław, Poland; phone: (48 71) 320 5435; fax: (48 71) 321 1567, e-mail: hutny@star.ar.wroc.pl
}

Abbreviations: VDAC, voltage dependent anion channels; Glc-6-P, glucose 6-phosphate; BSA, bovine serum albumin; SDS, sodium dodecyl sulfate. 
larly high levels in brain [2] where it plays an important role in regulating the rate of cerebral glucose (Glc) metabolism [3]. The major portion (> 80\%) of the hexokinase activity in brain is associated with mitochondria [4-9]. This physical proximity is thought to provide the basis for functional interaction between Glc phosphorylation by hexokinase and intramitochondrial ATP production by oxidative phosphorylation, with resulting coordination of the glycolytic and oxidative phases of Glc metabolism [10, 11]. The outer mitochondrial membrane protein responsible for specific interaction with hexokinase was originally isolated as the "hexokinase binding protein" [12] but quickly shown $[13,14]$ to be identical with porin (also called VDAC), the protein which forms the transmembrane channel by which metabolites enter or exit the mitochondria [15]. It thus appears that hexokinase is directly associated with the physical structure, the pore, by which substrate ATP may be delivered from its intramitochondrial source.

With mitochondria from rat brain, binding of hexokinase is readily reversible, with about $90 \%$ of the enzyme being released by incubation of the mitochondria with $\mathrm{mmol} / \mathrm{l}$ levels of Glc-6-P [16], the product of the hexokinase reaction. However, this is not generally true for mitochondrial hexokinases from brain of other species, with a maximum of about: $60 \%$, $40 \%$, and $20 \%$ of the hexokinase being released by Glc-6-P with mitochondria from rabbit, bovine, and human brain [16], respectively. The hexokinase that is not released with Glc-6-P can be solubilized by further treatment of the mitochondria with high salt concentrations [16, 17]. Previous work [16, 18] has led to the conclusion that this difference in sensitivity to release by Glc-6-P does not result from intrinsic differences in the hexokinase itself, but rather, reflects the existence of two different kinds of binding sites for the enzyme. Thus, Type A sites are defined as mitochondrial sites from which the enzyme is released by the action of Glc-6-P, while Type $B$ sites are those at which hexokinase remains bound even in the presence of Glc-6-P. In these terms, the Type A:Type $B$ ratio of binding sites is approximately $90: 10,60: 40,40: 60$, and $20: 80$ for mitochondria from rat, rabbit, bovine, and human brain, respectively.

Given the well-known heterogeneity of the mitochondrial population in brain [19-22], one obvious possibility was that the Type A and Type $B$ sites might reside on different subpopulations of brain mitochondria. However, this is not the case, with both Type A and Type B sites shown to coexist on the same mitochondria [18]. Isoforms of porin have also been reported [14, 15, 23], and thus another possibility was that Type A and Type B sites might correspond to involvement of different isoforms of porin in binding of hexokinase. However, this also appears not to be the case since rat and bovine brain mitochondria, while differing markedly in ratio of Type A:Type $B$ sites, were indistinguishable in their porin isoform content [18]. Thus, the molecular basis for the occurrence of these distinct types of hexokinase binding sites has remained unclear. The present study suggests that the phospholipid environment may play a major role in determining the Type A-Type B character of the mitochondrial binding site for hexokinase.

\section{MATERIALS AND METHODS}

Materials. Bovine brain was obtained at the Meats Laboratory (Michigan State University, U.S.A.), and transported to the laboratory on ice. Gross regions of white matter and larger blood vessels were dissected away, and the tissue was cut into smaller portions and stored in liquid nitrogen. Brains from rats, rabbits, and guinea pigs were obtained immediately after sacrifice of the animals and stored in liquid nitrogen, as in previous studies from this laboratory [16, 18]. Triton X-114, phospholipase C (catalog No. P-7633), and phospholipids were obtained from Sigma Chemical Co. (St. Louis, MO, U.S.A.), while 
Triton X-100 was from Research Products International (Elk Grove Village, IL, U.S.A.). Yeast Glc-6-P dehydrogenase was the product of Roche Molecular Biochemicals (Indianapolis, IN, U.S.A.). The bicinchoninic acid reagent (BCA) Reagent and bovine serum albumin (BSA) standard for protein assays were purchased from Pierce Chemical Co. (Rockford, IL, U.S.A.). AffiGel Blue was from BioRad Laboratories (Richmond, CA, U.S.A.) and silica gel 60 plates for thin-layer chromatography were products of Merck (Darmstadt, Germany).

Assay of hexokinase activity and protein. Hexokinase activity was determined using a previously described [24] spectrophotometric assay; Glc-6-P formation was coupled to $\mathrm{NADPH}$ production, monitored at $340 \mathrm{~nm}$, in the presence of excess Glc-6-P dehydrogenase. With samples containing Triton X-114, the assay mixtures were preincubated for approximately 2 min (to allow dissolution of the Triton X-114) before initiation of the reaction by addition of ATP.

Protein was determined by the bicinchoninic acid method, with BSA as standard.

Preparation of brain mitochondria. Mitochondria were prepared as described previously [25]. Briefly, brains were homogenized in $0.25 \mathrm{M}$ sucrose and a crude mitochondrial fraction was obtained by standard centrifugal fractionation. The crude mitochondrial pellet was resuspended in $0.25 \mathrm{M}$ sucrose, layered on $10 \mathrm{ml}$ of $1.2 \mathrm{M}$ sucrose, and centrifuged for 90 min at 35000 r.p.m. in a Beckman SW27 rotor. The mitochondrial pellets were resuspended in $0.25 \mathrm{M}$ sucrose to give a protein concentration of approx. $10 \mathrm{mg} / \mathrm{ml}$; the specific activity of mitochondrial hexokinase was 0.4-0.8 u/mg mitochondrial protein. Mitochondrial preparations were divided into convenient aliquots and stored at $-80^{\circ} \mathrm{C}$.

Solubilization of mitochondrial hexokinase with Glc-6-P and with high salt. Mitochondria were diluted to a concentration of $0.2 \mathrm{mg}$ mitochondrial protein $/ \mathrm{ml}$ with $0.25 \mathrm{M}$ sucrose, $1.2 \mathrm{mM}$ Glc-6-P, and $10 \mathrm{mM}$ Hepes,
$\mathrm{pH}$ 8.2. After incubation for $30 \mathrm{~min}$ at room temperature, the suspensions were centrifuged at $5^{\circ} \mathrm{C}$ for $10 \mathrm{~min}$ at $20000 \times \mathrm{g}$. The supernatants contained the hexokinase solubilized with Glc-6-P, i.e, hexokinase which had been bound at Type A sites.

To obtain hexokinase bound to Type B sites, bovine brain mitochondria were first treated with Glc-6-P, as above. The mitochondria, now containing only hexokinase bound to Type B sites, were resuspended in $0.25 \mathrm{M}$ sucrose, 2 $\mathrm{mM} \mathrm{MgCl}_{2}, 0.8 \%$ (v/v) Triton X-100, $10 \mathrm{mM}$ Hepes, $\mathrm{pH} 8.2$, to give a protein concentration of approx. $1.5 \mathrm{mg} / \mathrm{ml}$. After $5 \mathrm{~min}$ on ice, the suspension was centrifuged at $20000 \times \mathbf{g}$ for $10 \mathrm{~min}$. The resulting pellet was resuspended in $0.25 \mathrm{M}$ sucrose, $0.15 \mathrm{M} \mathrm{NaCl}, 20 \mathrm{mM}$ thioglycerol, $1 \%$ (v/v) Triton X-100, $10 \mathrm{mM}$ Hepes, $\mathrm{pH}$ 8.2, to give a protein concentration of approx. $0.2 \mathrm{mg} / \mathrm{ml}$. The suspension was incubated on ice for $20 \mathrm{~min}$, with occasional stirring, then centrifuged at $20000 \times \boldsymbol{g}$ for 10 min. The supernatant contained the saltsolubilized hexokinase, previously bound to Type B sites.

Partial purification of hexokinase from Type $A$ and Type $B$ sites of bovine brain mitochondria. This was done using a modification of the previously described procedure for purification of Type I hexokinase from rat brain [24].

To the Glc-6-P-solubilized hexokinase (approx. $4 \mathrm{u}$ of activity) from bovine brain mitochondria (see above), thioglycerol and EDTA were added to final concentrations of $20 \mathrm{mM}$ and $0.5 \mathrm{mM}$, respectively, and the $\mathrm{pH}$ adjusted to 7.0 with phosphoric acid. The enzyme was applied to a small ( $2 \mathrm{ml}$ bed volume) of AffiGel Blue, equilibrated with $10 \mathrm{mM}$ Glc, $0.5 \mathrm{mM}$ EDTA, $20 \mathrm{mM}$ thioglycerol, $50 \mathrm{mM}$ Tris/Cl, $\mathrm{pH}$ 7.0. The column was washed extensively with this same buffer, then with buffer of the same composition but at $\mathrm{pH}$ 8.0. A final wash with reverse flow (i.e., upward through the column) was done with this same buffer at $\mathrm{pH}$ 8.0 but containing $20 \%$ (v/v) glycerol. The enzyme was eluted by continued reverse flow 
with the latter buffer, to which $1.5 \mathrm{mM}$ Glc-6-P was added. The eluted enzyme was concentrated in an Amicon ultrafiltration device with YM30 membrane, diluted 25-fold with $0.25 \mathrm{M}$ sucrose, and again concentrated. This process was repeated twice to provide the enzyme in $0.25 \mathrm{M}$ sucrose, essentially free from previous buffer components. Final yield was approximately 1 unit ( $25 \%$ of initial activity).

The hexokinase from Type B sites was purified by a similar procedure. The salt-solubilized enzyme (approx. 5 units), obtained as above, was dialyzed against $0.5 \mathrm{mM}$ EDTA, 10 $\mathrm{mM}$ Glc, $20 \mathrm{mM}$ thioglycerol, $10 \mathrm{mM}$ sodium phosphate, $\mathrm{pH}$ 7.0. The enzyme was then purified by chromatography on AffiGel Blue as described above. Final yield was approx. 2 units (40\% of initial activity).

Phase partitioning with Triton X-114. Mitochondrial constituents were partitioned between detergent (hydrophobic) and aqueous phases based on the procedure of Bordier [26]. Mitochondria were resuspended at a protein concentration of $0.5-1 \mathrm{mg} / \mathrm{ml}$ in $0.25 \mathrm{M}$ sucrose, $0.5 \mathrm{mM}$ EDTA, 1\% (v/v) Triton X-114, $20 \mathrm{mM}$ Hepes, $\mathrm{pH}$ 7.4. After incubation for 20 min on ice, with occasional mixing, the samples were centrifuged at $4^{\circ} \mathrm{C}$ and $100000 \times \mathbf{g}$ for $60 \mathrm{~min}$ in a Beckman Ti50 rotor. Aliquots $(120 \mu \mathrm{l})$ of the supernatant were transferred to prechilled $500 \mu \mathrm{l}$ microfuge tubes on ice; where indicated, $\mathrm{MgCl}_{2}$ was added. The samples were incubated for $2 \mathrm{~min}$ at $37^{\circ} \mathrm{C}$, resulting in development of turbidity as the detergent and aqueous phases separated. After centrifugation for $1 \mathrm{~min}$ at room temperature in a microfuge to resolve the aqueous and hydrophobic phases, the overlying aqueous layer was carefully removed. Following the protocol of Bordier [26], additional buffer and detergent were added so that the volume and composition of the two fractions were similar. Hexokinase content was then determined by activity assay and/or quantitative densitometry after SDS gel electrophoresis. For electrophoresis, proteins were precipitated by ad- dition of three volumes of cold $\left(-18^{\circ} \mathrm{C}\right)$ acetone and collected by centrifugation.

Electrophoresis and densitometry. SDS gel electrophoresis was done on $6.5-20 \%$ acrylamide gradient gels and the gels stained with Coomassie Blue, as previously described [27]. Gels were imaged with a GDS-2000 gel documentation system (UVP Inc., San Gabriel, CA, U.S.A.) and band intensity determined with the SW-2000 quantitative image analysis software provided by the manufacturer.

Extraction and analysis of mitochondrial lipids. Mitochondrial lipids were extracted by the procedure of Bligh and Dyer [28] and analyzed by two-dimensional thinlayer chromatography on silica gel plates using solvent "System A" of Rouser \& Fleischer [29]: 1st dimension, chloroform/methanol/ water (65:25:4, by vol.); 2nd dimension, n-butanol/acetic acid/water (60:20:20, by vol.). Identification of lipids was based on co-migration with authentic standards as well as detection with reagents specific for particular lipids [30]. Spots representing the most abundant lipids were scraped from the plates, the lipids eluted with chloroform, and samples analyzed by fast atom bombardment tandem mass spectrometry in the Michigan State University Mass Spectrometry Facility. This provided confirmation of the identity, as well as additional information about the nature of acyl groups present.

Enrichment of bovine brain mitochondria in specific phospholipids. Bovine brain mitochondria were enriched in particular phospholipids with minor modifications of the procedure of Parlo \& Coleman [31]. "Nude" Sephadex G-10 beads were prepared by washing the beads with chloroform/methanol (2: 1 , v/v) followed by drying under vacuum. Portions (100 mg) of the nude beads were placed in $25 \mathrm{ml}$ round bottom flasks and mixed with $5 \mathrm{ml}$ of a $1 \mathrm{mM}$ solution of the indicated phospholipid in chloroform/methanol $(2: 1$, $\mathrm{v} / \mathrm{v})$. The solvent was then removed on a ro- 
tary evaporator, leaving the phospholipid-coated Sephadex beads deposited as a thin film at the periphery of the flask. Mitochondrial suspension $(2 \mathrm{ml})$ containing 8-10 mg mitochondrial protein in $0.25 \mathrm{M}$ sucrose, $5 \mathrm{mM}$ Hepes, $\mathrm{pH}$ 7.4, was added. The flasks were incubated on ice with gentle shaking for $30 \mathrm{~min}$. The suspension was then layered over $30 \mathrm{ml}$ of $60 \%(\mathrm{w} / \mathrm{v})$ sucrose and centrifuged in a clinical centrifuge (approx. 400 $\times \boldsymbol{g}$ ) for $10 \mathrm{~min}$. Mitochondria were carefully harvested from the interface above the $60 \%$ sucrose, diluted with $0.25 \mathrm{M}$ sucrose and collected by centrifugation at $20000 \times \boldsymbol{g}$ for 10 min, and finally resuspended in $0.25 \mathrm{M}$ sucrose. Lipid extracts were examined by two-dimensional thin-layer chromatography, which confirmed enrichment (estimated 2- to 3-fold, based on intensity of the spot) in the indicated phospholipid.

Statistical analysis. Data were analyzed using GraphPAD Instat Version 1.13 (Graph Pad Software, San Diego, CA, U.S.A.).

\section{RESULTS}

\section{Partitioning of mitochondrial hexokinase between aqueous and hydrophobic phases}

Proportionally more white matter is in the brains of larger than in smaller animals, and this part of brain tissue is almost depleted of mitochondria. Thus to obtain comparable yield of mitochondria from brains of different origin, bigger chunks of white matter were removed from bovine brain before its storage in liquid nitrogen (see "Material and Methods"). This small difference in the treatment of bovine material did not change the hexokinase distribution between the A and B sites. Approximately $40 \%$ release of hexokinase by Glc-6-P was found by Kabir \& Wilson [18] in mitochondria prepared from the whole bovine brains. Very close values, usually between $36 \%$ and $43 \%$ were also observed in several experiments of this work (see Table 2). Such re- sult was expected, since only mitochondrially bound pool of hexokinase was analysed and most of the brain hexokinase is bound to mitochondria [4-9].

Solubilization of hexokinase bound to Type $B$ sites requires treatments that disrupt membrane integrity [16]. This suggested an intimate interaction between the enzyme and the outer mitochondrial membrane, more-or-less equivalent to that seen with "integral" membrane proteins [32]. Moreover, divalent cations have been shown to be important in mediating the interaction between hexokinase and the mitochondrial membrane [12, 17]. Based on these considerations, we decided to examine the partitioning of mitochondrial hexokinase between hydrophobic and aqueous phases using the method of Bordier [26], and the effects of divalent cations on that partitioning. This method takes advantage of the fact that Triton X-114 is miscible with water at low temperatures, but condenses into a discrete phase after warming to moderate temperature (about $30^{\circ} \mathrm{C}$ ). Thus, solubilization of membranes with Triton X-114 in aqueous buffer at low temperatures, followed by a brief incubation at higher temperature, results in development of discrete aqueous and detergent phases, with "integral” membrane proteins being selectively partitioned into the hydrophobic phase while hydrophilic (peripheral) membrane proteins are found in the aqueous phase [26].

As expected for a typical water-soluble protein [26], the purified Type 1 hexokinase from rat brain [24] was largely confined to the aqueous phase after partitioning in either the absence or presence of $2 \mathrm{mM} \mathrm{MgCl} 2$ (Table 1). The same was true for the hexokinase obtained from bovine brain mitochondria by elution with either Glc-6-P (from Type A sites) or high salt (from Type B sites). When intact rat brain mitochondria, containing hexokinase bound predominantly to Type A sites, were subjected to the partitioning procedure, with or without addition of $\mathrm{MgCl}_{2}$, the hexokinase again behaved as a hydrophilic protein, with 
Table 1. Partitioning of hexokinase between aqueous and hydrophobic (Triton X-114) phases

\begin{tabular}{|c|c|c|c|}
\hline \multirow[b]{2}{*}{ Sample partitioned } & \multirow[b]{2}{*}{ Originally bound to } & \multicolumn{2}{|c|}{ Percent of hexokinase activity in aqueous phase ${ }^{a}$} \\
\hline & & No $\mathrm{MgCl}_{2}$ & $+2 \mathrm{mM} \mathrm{MgCl}{ }_{2}$ \\
\hline $\begin{array}{l}\text { Purified hexokinase from } \\
\text { rat brain }\end{array}$ & Predominantly Type A sites & 91 & 92 \\
\hline $\begin{array}{l}\text { Purified Glc-6-P-solubilized } \\
\text { hexokinase, bovine brain }\end{array}$ & Type A sites & 94 & 88 \\
\hline $\begin{array}{l}\text { Purified salt-solubilized } \\
\text { hexokinase, bovine brain }\end{array}$ & Type B sites & 86 & 83 \\
\hline Rat brain mitochondria ${ }^{b}$ & Predominantly Type A sites & $85(76)$ & $82(77)$ \\
\hline Bovine brain mitochondria ${ }^{\mathrm{b}}$ & Type A and Type B sites & $87(80)$ & $37(39)$ \\
\hline
\end{tabular}

${ }^{\mathrm{a}}$ Hexokinase activity was determined for both aqueous and detergent phases; total activity recovered was $>95 \%$ of initial activity. ${ }^{\mathrm{b}}$ For these samples, the distribution between aqueous and detergent phases was also determined by densitometric analysis after SDS gel electrophoresis, yielding the values shown in parenthesis.

the enzyme being found predominantly in the aqueous phase. This was also true when isolated bovine brain mitochondria, containing hexokinase bound to both Type A and Type B sites, were partitioned in the absence of divalent cation. But when partitioning of bovine brain mitochondria was done in the presence of $2 \mathrm{mM} \mathrm{MgCl}$, there was a marked decrease in the amount of hexokinase found in the aqueous phase, with corresponding increase in the amount in the hydrophobic phase.

The proteins present in aqueous and detergent phases after partitioning of rat or bovine brain mitochondria were examined by SDS gel electrophoresis, and hexokinase band was identified by comparison with purified rat brain hexokinase used as a marker (Fig. 1). As in previous studies [33], a slight difference in mobility of hexokinase from bovine and rat mitochondria has been noticed. In a separate experiment the identity of the band designated as type I hexokinase was confirmed in both bovine and rat SDS extracts by immunoblotting with antihexokinase I (not shown). Electrophoresis permitted several observations of interest. First, it was apparent that the overall protein composition of bovine and rat brain was similar; most of the components seen in rat brain mitochondria had their counterparts in bovine brain mitochondria, and vice versa. This is, of course, not unexpected since mitochondria, whatever their source, must contain the basic set of proteins required for common mitochondrial functions. Second, despite the fact that addition of $\mathrm{MgCl}_{2}$ did not affect the partitioning of rat brain mitochondrial hexokinase (Table 1), it was clear that partitioning of several other rat brain mitochondrial proteins into the hydrophobic phase was increased by the inclusion of

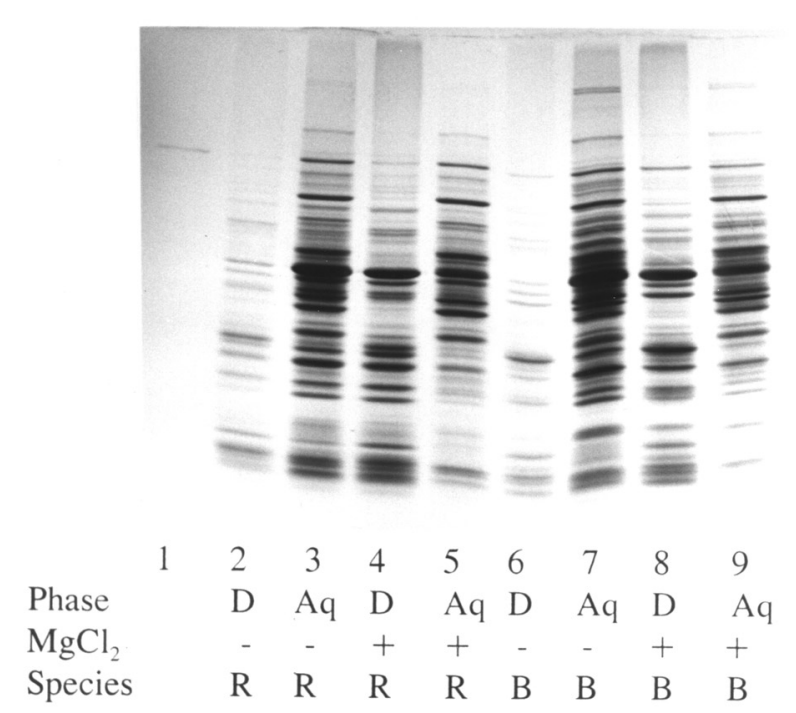

Figure 1. Electrophoretic analysis of proteins present in detergent and aqueous phases after Triton X-114 partitioning of rat and bovine brain mitochondria.

Lane 1 is purified rat brain hexokinase [24], run as a marker. Lanes 2-9 contained detergent (D) or aqueous (Aq) phases after Triton X-114 partitioning [26] of rat (R) or bovine (B) brain mitochondria in the absence (-) or presence (+) of $2 \mathrm{mM} \mathrm{MgCl}_{2}$, as described in Methods. 
this salt, while other protein components, together with hexokinase, remained in the aqueous phase in either the absence or presence of $\mathrm{MgCl}_{2}$. Thus, the effect of $\mathrm{MgCl}_{2}$ was selective. Third, a similar effect was seen with bovine brain mitochondria, with inclusion of $\mathrm{MgCl}_{2}$ during the partitioning resulting in a selective shift of several proteins into the hydrophobic phase. Moreover, several of the bovine brain mitochondrial proteins showing increased partitioning into the detergent phase were virtually identical in mobility to rat brain mitochondrial proteins showing similar behavior; these are reasonably presumed to represent homologs, and would thus indicate that these proteins show similar partitioning behavior, whether derived from rat or bovine brain mitochondria. Thus, the difference in partitioning of hexokinase is not reflective of a general difference in partitioning behavior of proteins from rat and bovine brain mitochondria.

It should be apparent that partitioning of mitochondrial proteins must be dependent on factors more complex than a simple division into "integral" or "peripheral" proteins. In the study of Bordier [26], partitioning of integral membrane proteins into the detergent phase was attributed to a "hydrophobic domain" expected to be included within the structure of such proteins and responsible for interaction with the hydrophobic core of the membrane. The hydrophobic domain would, of course, be intrinsic and hence partitioning into the detergent phase expected to be independent of the presence or absence of $\mathrm{MgCl}_{2}$. This is in contrast to the observed $\mathrm{MgCl}_{2}$-induced shift of several proteins (including the hexokinase of bovine, but not rat, brain mitochondria) to the detergent phase.

The fact that an increased proportion of several proteins were found to partition into the detergent phase in the presence of $\mathrm{MgCl}_{2}$ suggested the possibility that inclusion of $\mathrm{MgCl}_{2}$ had induced the formation of an aggregate. Indeed, extended ultracentrifugation (100000 $\times \boldsymbol{g}$ for $30 \mathrm{~min}$ at room temperature in a Beckman Airfuge) of the detergent phase gave a small pellet. Analysis of the pellet and the overlying detergent phase by SDS gel electrophoresis showed them to be indistinguishable in protein composition (and as shown in Fig. 1), with approximately equal amounts of protein being found in both pellet and "soluble" in the detergent phase. Thus, it would appear that at least some aggregation may be associated with the observed partitioning behavior.

\section{$\mathrm{Mg}^{++}$-induced partitioning into the deter- gent phase is inversely related to the pro- portion of Type A sites present in the mito- chondria}

The proportion of hexokinase bound to Type A sites of brain mitochondria increases in the order of bovine < rabbit < guinea pig < rat brain (Table 2). This is in agreement with previous findings [16] except that the modest difference between rabbit and guinea pig brain mitochondria had not been noted in the earlier work. When partitioning of hexokinase bound to these mitochondria was examined, it was found that the $\mathrm{Mg}^{++}$-induced partitioning into the detergent phase decreased in a similar order (Table 2), with values for bovine and rat brain mitochondria lying at the extremes while values for guinea pig and rabbit (not significantly different from each other) were intermediate.

\section{$\mathrm{Mg}^{++}$-induced partitioning into the deter- gent phase is not associated with occupancy of Type B sites}

The results in Table 2 suggested the possibility that it was hexokinase bound at Type B sites that was selectively partitioned into the detergent phase in the presence of $\mathrm{Mg}^{++}$. Thus, as the proportion of hexokinase bound to Type A sites increased, and the proportion occupying Type B sites correspondingly decreased, decreased partitioning into the detergent phase was observed. If this were the case, then elution of the enzyme bound to Type A 
sites prior to partitioning (i.e., all of the hexokinase left on the mitochondria would be occupying Type B sites) should result in a marked increase in the hexokinase found in the detergent phase. However, when partitioning was done with bovine brain mitochondria which had been depleted of hexokinase bound at Type A sites by incubation with Glc-6-P, the $\mathrm{Mg}^{++}$-induced increase in partitioning into the detergent phase was $27 \pm 3 \%$ parting sufficient hydrophobic character to lead to significant partitioning into the detergent phase. Previous work [34] implicating membrane phospholipids in the hexokinasemitochondrial interaction prompted experiments to examine possible involvement of phospholipids in the observed partitioning behavior.

In an initial experiment, rat and bovine brain mitochondria were solubilized with Tri-

Table 2. Type A sites and partitioning of brain mitochondria from different species

\begin{tabular}{lcc}
\hline & \multicolumn{2}{c}{ Hexokinase activity $^{\mathrm{a}}$} \\
\cline { 2 - 3 } Brain mitochondria & $\begin{array}{c}\text { \% solubilized with Glc-6-P } \\
\text { (Type A sites) }\end{array}$ & Mg $^{++}$-induced decrease in aqueous \\
phase $^{\mathrm{c}}$
\end{tabular}

${ }^{\mathrm{a}}$ Mean \pm S.D. for number of independent experiments shown in parenthesis. ${ }^{\mathrm{b}}$ Values for rabbit and guinea pig are significantly different with $P<0.01$. All other comparisons are significantly different with $P<0.001$. $^{\mathrm{c}}$ Values shown are percent of hexokinase found in the aqueous phase in the absence of $\mathrm{MgCl}_{2}$ minus the percent found in the aqueous phase after partitioning in the presence of $\mathrm{MgCl}_{2}$. Values for rabbit and guinea pig are not significantly different $(P>0.05)$. Values for bovine and guinea pig are significantly different with $P<0.05$. All other comparisons are significantly different with $P<0.001$.

(for four independent experiments), not significantly different from the value of $30 \pm 3 \%$ (Table 2) obtained with bovine brain mitochondria as isolated (i.e., with hexokinase occupying both Type A and Type B sites). Thus, the partitioning results depend on the relative proportions of Type A and Type B sites present on the mitochondria, but are not determined by whether the hexokinase being partitioned is actually occupying Type A or Type B sites.

\section{Partitioning of hexokinase into the deter- gent phase is dependent on phospholipids}

Since hexokinase itself did not partition into the detergent phase (Table 1), these results suggested that partitioning behavior was dependent on $\mathrm{Mg}^{++}$-induced interaction of hexokinase with one or more hydrophobic components of bovine brain mitochondria, im- ton $\mathrm{X}-114$, as for a standard partitioning experiment (see Methods). However, prior to the brief incubation at $37^{\circ} \mathrm{C}$ to produce the phase separation, 50 milliunits of phospholipase $\mathrm{C}$ were added and the samples were incubated for $3 \mathrm{~h}$ at $5-10^{\circ} \mathrm{C}$; control samples were incubated in the same way, but without addition of phospholipase C. The samples were then warmed to $37^{\circ} \mathrm{C}$ and partitioning continued as usual. The slight $\mathrm{Mg}^{++}$-induced increase in partitioning of rat brain mitochondrial hexokinase was virtually unchanged by phospholipase $\mathrm{C}$ treatment, being $7 \%$ and $4 \%$ in the control and treated samples, respectively; these values are in good agreement with those seen in standard partitioning experiments (Table 2). In contrast, the corresponding values for bovine brain mitochondria were $26 \%$ (again in agreement with results in standard partitioning protocol - Table 2) and 5\% for control and treated samples, respectively. 
The effect of phospholipase was dependent on both time of treatment and amount of phospholipase used. Thus, after solubilization of bovine brain mitochondria with Triton $\mathrm{X}-114$, as above, the $\mathrm{Mg}^{++}$-induced increase in partitioning into the detergent phase was $24 \%$ in the control (no phospholipase added) and $15 \%$ and $7 \%$ after 1 and $2 \mathrm{~h}$, respectively, treatment with 100 milliunits of phospholipase C. In a similar experiment, the detergentsolubilized bovine brain mitochondria were incubated for $1 \mathrm{~h}$ with $0,50,100$, or 200 milliunits of phospholipase $\mathrm{C}$; subsequent partitioning showed the $\mathrm{Mg}^{++}$-induced increase in partitioning into the detergent phase was 25 , 14,7 and $4 \%$, respectively.

Phospholipase treatment of intact mitochondria produced similar results. Thus, bovine brain mitochondria were incubated $\left(5-10^{\circ} \mathrm{C}\right.$, $30 \mathrm{~min}$ ) with 0 , 40, or 200 milliunits of phospholipase $\mathrm{C}$, then collected by centrifugation. Assay of the supernatant revealed $<5 \%$ of the hexokinase had been released during phospholipase treatment. The mitochondrial pellets were resuspended and a portion used for partitioning by the usual protocol. The $\mathrm{Mg}^{++}$-induced increase in partitioning into the detergent phase was 31,26 , and $14 \%$ for the mitochondria treated with 0,40 , and 200 milliunits of phospholipase $\mathrm{C}$, respectively. Lipids were extracted from another portion of the phospholipase-treated mitochondria; analysis by 2-dimensional thin-layer chromatography confirmed substantial decrease in all phospholipid components.

In all of the above experiments, proteins present in aqueous and detergent phases were examined by SDS gel electrophoresis. Control samples (no phospholipase added) gave results as shown in Fig. 1, lanes 6-9. Phospholipase treated samples were, qualitatively, identical to controls, but it was evident that phospholipase treatment resulted in a decreased partitioning of all protein components into the detergent phase, i.e., the effects of phospholipase were not selective for hexokinase.

\section{Comparison of the phospholipid composi- tion of bovine and rat brain mitochondria}

Lipids extracted from bovine and rat brain mitochondria were examined by 2-dimensional thin-layer chromatography (Fig. 2). The

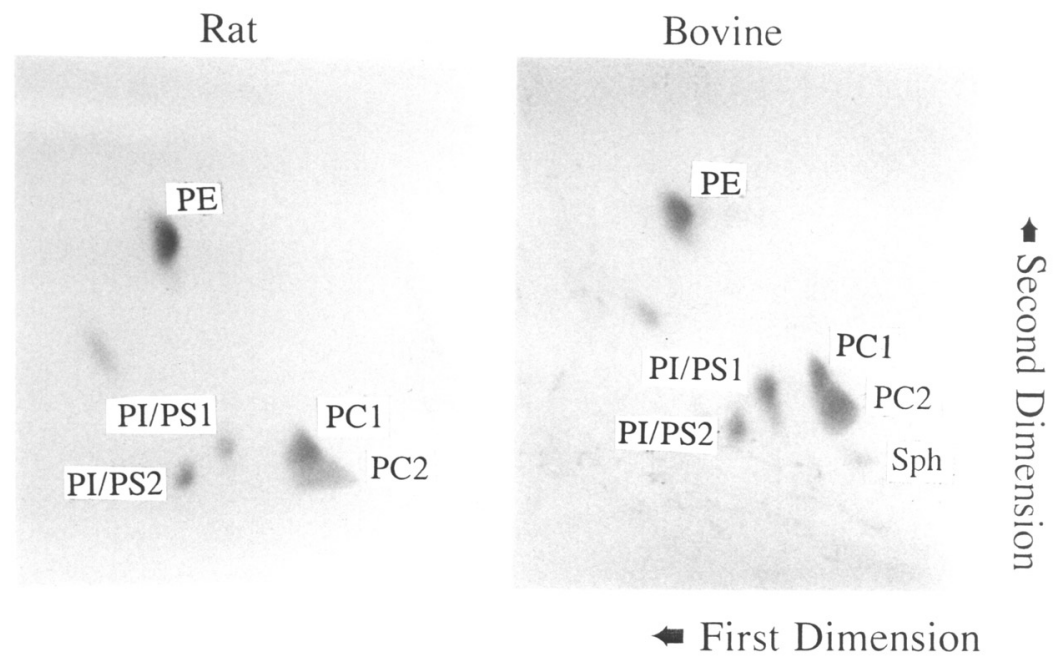

Figure 2. Thin-layer chromatographic analysis of lipids from rat and bovine brain mitochondria.

Lipids were extracted and analyzed by thin-layer chromatography as described in Methods. Prominent components were phosphatidylethanolamine (PE), two spots (PC1 and PC2) identified as phosphatidylcholine with differing acyl group composition (see text), and two spots (PI/PS1 and PI/PS2), both of which contained phosphatidylinositol as well as phosphatidylserine. Sphingomyelin ( $\mathrm{Sph}$ ) was detected in extracts from bovine brain mitochondria, but not rat brain mitochondria. Both types of mitochondria also contained sulfatide (unmarked spot, upper left from "PI/PS1" label). 
same major lipid components were detected in both kinds of mitochondria but there were some differences, reproducibly seen in several such comparisons.

First, phosphatidylcholine was partially resolved into two overlapping spots, designated PC1 and PC2 in Fig. 2. PC1 was consistently more intense in extracts from rat brain mitochondria, while the more diffuse, slower moving PC2 was more intense with bovine brain mitochondria. Mass spectrometric analysis confirmed identification as phosphatidylcholine, but revealed that the PC1 and PC2 differed in fatty acid composition. PC1 contained primarily stearoyl (18:0) and 20 carbon acyl groups, the latter with 1, 2, or 4 double bonds. In contrast, PC2 contained shorter acyl groups, primarily palmitoyl $(16: 0)$, with some stearoyl $(18: 0)$, oleoyl $(18: 1)$, and myristoyl (14:0) groups.

Secondly, sphingomyelin (Sph in Fig. 2) was consistently detected as a minor component in extracts from bovine brain mitochondria, but was barely detectable in rat brain mitochondrial extracts.

Finally, two spots (labelled PI/PS1 and PI/ PS2 in Fig. 2) migrated in the region expected for phosphatidylinositol and phosphatidylserine. The relative amounts of PI/PS1 and
PI/PS2 were comparable in rat brain mitochondria, while PI/PS2 < PI/PS1 in bovine brain mitochondria. Although well resolved, both spots were ninhydrin-positive, indicating the presence of phosphatidylserine but this could not be confirmed by mass spectrometry. Mass spectrometry did confirm the presence of phosphatidylinositol, with stearoyl (18:0) and arachidonoyl $(20: 4)$ as primary acyl groups, in both spots. Commercially obtained phosphatidylserine and phosphatidylinositol both gave a single large spot that encompassed both the PI/PS1 and PI/PS2 regions. The basis for resolution of the mitochondrial phospholipids into two spots, with both phosphatidylinositol and phosphatidylserine being present in both spots, is unclear.

\section{Solubilization of mitochondrial hexokinase with Triton X-100, Triton X-114, and digitonin}

The mitochondrial hexokinases from bovine, rabbit, guinea pig, and rat differed in their susceptibility to solubilization by Triton X-100 and Triton X-114 (Table 3), with the bovine enzyme being most resistant, the rat brain enzyme most susceptible, and the rabbit and guinea pig enzymes intermediate. In each

Table 3. Solubilization of mitochondrial hexokinase with Triton X-114, Triton X-100, or digitonin

\begin{tabular}{|c|c|c|c|c|c|}
\hline \multirow{2}{*}{ Detergent added } & \multirow{2}{*}{$\begin{array}{l}\text { Concentration (mg/mg } \\
\text { mitochondrial protein) }\end{array}$} & \multicolumn{4}{|c|}{$\%$ of hexokinase solubilized with brain mitochondria from } \\
\hline & & Bovine & Rabbit & Guinea pig & Rat \\
\hline None & - & $4 \pm 2$ & $8 \pm 2$ & $8 \pm 3$ & $9 \pm 2$ \\
\hline \multirow[t]{4}{*}{ Triton X-114 } & 0.2 & $10 \pm 2$ & $19 \pm 3$ & $18 \pm 2$ & $35 \pm 5$ \\
\hline & 0.35 & $29 \pm 1$ & $48 \pm 7$ & $47 \pm 2$ & $71 \pm 5$ \\
\hline & 0.5 & $68 \pm 3$ & $87 \pm 4$ & $85 \pm 1$ & $92 \pm 4$ \\
\hline & 1.0 & $88 \pm 4$ & $91 \pm 2$ & $95 \pm 1$ & $96 \pm 1$ \\
\hline \multirow[t]{3}{*}{ Triton X-100 } & 0.4 & $30 \pm 4$ & $47 \pm 7$ & $43 \pm 10$ & $59 \pm 8$ \\
\hline & 2 & $50 \pm 4$ & $70 \pm 4$ & $69 \pm 11$ & $78 \pm 6$ \\
\hline & 50 & $89 \pm 1$ & $93 \pm 2$ & $95 \pm 3$ & $96 \pm 3$ \\
\hline \multirow[t]{3}{*}{ Digitonin } & 0.1 & $6 \pm 2$ & $13 \pm 1$ & $12 \pm 4$ & $13 \pm 3$ \\
\hline & 0.8 & $7 \pm 2$ & $14 \pm 3$ & $12 \pm 1$ & $17 \pm 6$ \\
\hline & 1.6 & $13 \pm 2$ & $19 \pm 3$ & $18 \pm 5$ & $30 \pm 4$ \\
\hline
\end{tabular}

${ }^{\mathrm{a}}$ Values shown are mean \pm S.D. for nine samples with no added detergent, and for three samples at each concentration of detergent. 
case, Triton X-114 was more effective than Triton X-100. These detergents differ in their relative hydrophobic character, with Triton X-100 containing an average of 9.5 hydrophilic oxyethylene groups per (hydrophobic) octylphenol headgroup, while the more hydrophobic Triton X-114 contains only 7-8 oxyethylene groups per octylphenol (Product Information Sheet, Sigma Chemical Co.).

Confirming previous results [10, 35, 36], brain mitochondrial hexokinase was rather resistant to solubilization by digitonin (Table 3). Again, there were differences seen with brain mitochondria from different species, with hexokinase of bovine brain mitochondria being the most resistant, the hexokinase of rat brain mitochondria most susceptible, and hexokinase of guinea pig and rabbit mitochondria intermediate in susceptibility to solubilization with digitonin. As in previous studies [10, 35, 36], a maximum of about $30 \%$ of the hexokinase could be solubilized from rat brain mitochondria; with bovine brain mitochondria, a maximum of about $15 \%$ could be solubilized at the highest digitonin concentrations used. In contrast, $80-90 \%$ of the adenylate kinase, a marker for the intermembranal space, was released from both bovine and rat brain mitochondria with only about $0.5 \mathrm{mg}$ digitonin/mg protein ([10, 35, 36], and data not shown). Digitonin is thought to exert its membrane-disrupting effect through complexing with cholesterol [37]. Thus these results are consistent with the suggestion [35] that the majority of the hexokinase on brain mitochondria is associated with cholesteroldeficient regions of the outer membrane thought to be located at contact sites [35, 38-40].

\section{Effect of enrichment of mitochondrial mem- branes in specific phospholipids}

Bovine brain mitochondria were selectively enriched in specific phospholipids and then treated with Glc-6-P to release hexokinase from Type A sites. Subsequent re-incubation of the mitochondria with Glc-6-P evoked no further Glc-6-P-dependent release - as expected, since the enzyme had previously been removed from Type A sites. Enrichment of the mitochondria in the indicated phospholipids had, at most, a marginal effect on solubilization of hexokinase in either the absence or presence of Glc-6-P (Table 4). However, if the enriched mitochondria were first incubated at $37^{\circ} \mathrm{C}$, solubilization in both the absence and presence of Glc-6-P was enhanced by enrichment in acidic phospholipids while enrichment in phosphatidylcholine or phosphatidylethanolamine was without significant effect. These results are consistent with previ-

Table 4. Enrichment in acidic phospholipids, but not phosphatidylcholine or phosphatidylethanolamine, alters binding of hexokinase to bovine brain mitochondria ${ }^{\text {a }}$

\begin{tabular}{lcccc}
\hline & \multicolumn{2}{c}{ No incubation at $37^{\circ} \mathrm{C}$} & \multicolumn{2}{c}{ After 4 h incubation at $37^{\circ} \mathrm{C}$} \\
\cline { 2 - 4 } & \% hexokinase solubilized & \% hexokinase solubilized \\
\hline Mitochondria enriched in & No Glc-6-P & Plus Glc-6-P & No Glc-6-P & Plus Glc-6-P \\
No exogenous lipids & $7 \pm 2$ & $9 \pm 3$ & $9 \pm 0$ & $19 \pm 5$ \\
Phosphatidylcholine & $6 \pm 2$ & $8 \pm 2$ & $10 \pm 2$ & $16 \pm 4$ \\
Phosphatidylethanolamine & $6 \pm 1$ & $8 \pm 1$ & $9 \pm 3$ & $15 \pm 4$ \\
Phosphatidylserine & $11 \pm 1$ & $15 \pm 2$ & $28 \pm 8$ & $40 \pm 6$ \\
Phosphatidylinositol & $13 \pm 8$ & $14 \pm 5$ & $28 \pm 4$ & $42 \pm 12$ \\
\hline
\end{tabular}

a Bovine brain mitochondria were enriched in the indicated phospholipids; as a control ("no exogenous lipids"), mitochondria were subjected to the same enrichment protocol but without addition of exogenous lipids. The mitochondria were treated with Glc-6-P (as in Methods) to elute hexokinase bound at Type A sites, then either maintained on ice or incubated at $37^{\circ} \mathrm{C}$ for $4 \mathrm{~h}$. Aliquots were then incubated for $20 \mathrm{~min}$ at room temperature either with or without addition of $1.2 \mathrm{mM}$ Glc-6-P. Following centrifugation, the percent of total hexokinase activity in solubilized form (i.e., in the supernatant) was determined. ${ }^{b}$ Values shown are mean \pm S.D.; $\mathrm{n}=5$ for control samples (no exogenous lipids); $\mathrm{n}=3$ for all others. 
ous work [34] implicating acidic phospholipids as significant factors influencing the hexokinase-mitochondrial membrane interaction, and suggest that reorganization of phospholipid components (during the incubation at $37^{\circ} \mathrm{C}$ ) was required for manifestation of this effect.

Another interesting aspect of the results shown in Table 4 should be noted. As mentioned above, if control (i.e., not enriched in exogenous lipids) were pre-treated with Glc-6-P to elute hexokinase from the Type A sites and then kept at $0^{\circ} \mathrm{C}$ before a second incubation with Glc-6-P, no further Glc-6-P-dependent release was seen. However, if the mitochondria were incubated at $37^{\circ} \mathrm{C}$ prior to the second treatment with Glc-6-P, there was a highly significant $(P<0.003)$ increase in Glc-6-P-dependent solubilization but no effect on solubilization in the absence of Glc-6-P. In other words, some redistribution of the enzyme, initially bound at Type B sites, had occurred during the incubation at $37^{\circ} \mathrm{C}$. Whether this resulted from spontaneous dissociation of the enzyme from Type B sites and rebinding at Type A sites, or from an actual conversion of some of the Type B sites to Type A sites cannot be determined from these results. In either case, these results suggest a dynamic relationship between Type $\mathrm{A}$ and Type B sites.

\section{DISCUSSION}

The present study has extended previous work $[16,18]$ indicating notable differences in the binding of hexokinase to brain mitochondria from different species. It appears that the lipid environment may play a significant role in determining the properties of the binding site for hexokinase in mitochondrial membrane. Authors' intention was to use the outer mitochondrial membrane as a basic material for experiments of this work. But the separation methods of mitochondrial membranes from brain tissue are still not developed enough to obtain the purified product. For unknown reason the "swelling and shrinking" procedure, which give excellent results with other tissues, does not work well with brain mitochondria. Also alternative procedures $[35,41]$ do not provide a satisfactory enrichment of standard outer membrane markers, and our attempts of their improvement were unsuccessful. Because of these technical problems, we limited the analysis to whole mitochondria.

A general resistance of mitochondrially bound hexokinase to solubilization by digitonin was a common characteristic for all species examined, yet there were differences implying that the relative cholesterol content and hence susceptibility to disruption by digitonin [37] - varied with species. It is clear that this does not represent a general resistance of the outer mitochondrial membrane to digitonin since the membrane is disrupted, as indicated by release of adenylate kinase from the intermembranal space as well as solubilization of other outer mitochondrial membrane enzymes [10, 35, 36] at digitonin concentrations that cause release of only marginal amounts of hexokinase. That "lipid domains”, i.e., regions of varying lipid composition, may exist within a membrane was first proposed by Karnovsky et al. [42] and is now a generally accepted principle of membrane structure [43, 44]. Indeed, formation of such domains may be induced by membrane proteins [45]. The heterogeneous response of various outer mitochondrial membrane enzymes to solubilization by digitonin implies that they reside in domains differing in relative content of cholesterol, and likely other lipids. The apparently cholesterol-deficient regions, in which most of the mitochondrially bound hexokinase is located, are suggested to be contact sites [35, 38-40], i.e., regions of intimate contact, perhaps fusion, between the inner and outer mitochondrial membranes.

Approximately $70 \%$ of the hexokinase bound to rat brain mitochondria $([10,35,36]$, and present work) was resistant to solubilization 
by digitonin, while this was about $90 \%$ for the bovine mitochondrial hexokinase, with mitochondrial hexokinases from guinea pig and rabbit brain falling between these extremes. While the Triton detergents were much more effective than digitonin, an analogous order was seen in the relative susceptibility to solubilization; in all cases Triton X-114 was more effective (on a mg detergent per mg protein basis) that the more hydrophilic Triton $\mathrm{X}-100$. These results thus imply that solubilization of hexokinase requires access to a highly hydrophobic cholesterol-poor domain, with relative accessibility and cholesterol content decreasing in the order rat > guinea pig ? rabbit > bovine.

It is clear that phospholipid composition, particularly content of acidic phospholipids, can markedly affect the interaction of hexokinase with mitochondria ([34], and present work). However, as shown by the results in Table 4 , incorporation of acidic phospholipids into the mitochondrial membranes is not, in itself, sufficient to alter the hexokinase-membrane interaction. Rather, the effects of altered phospholipid content became evident only after preincubation of the enriched mitochondria at elevated temperature $\left(37^{\circ} \mathrm{C}\right)$. This suggests that the effects on hexokinase binding are seen only after a reorganization of the phospholipids, randomly incorporated during the enrichment process, into domains closely associated with the hexokinase binding sites. We hypothesize that Type A and Type B sites reside within lipid domains of differing phospholipid composition. In particular, we suggest that the lipid environment of the Type A site may be enriched in acidic phospholipids which have previously been shown [34] to enhance susceptibility of the mitochondrially bound enzyme to solubilization with Glc-6-P. No gross differences in phospholipid composition, including the acidic phospholipids, were detected in mitochondria from rat and bovine brain, despite the fact that these mitochondria differ considerably in relative proportion of Glc-6-P-sensitive (Type A) sites present.
However, this does not preclude the possibility that the organization of these phospholipids may differ, with increased sequestration of acidic phospholipids into "Type A" lipid domains leading to increased proportion of the hexokinase bound in a Glc-6-P-sensitive manner. What would prompt such different lipid organization in membranes that did not differ markedly in overall lipid composition is, of course, a major question, for which we presently cannot suggest an answer.

As noted above, the $\mathrm{Mg}^{++}$-dependent partitioning into the Triton X-114 phase is not correlated with occupancy of Type B sites but rather with the relative proportion of Type $B$ sites present on the mitochondria subjected to the partitioning regimen. Since the partitioning behavior does not depend on whether the hexokinase is associated with both Type $\mathrm{A}$ and Type B sites (bovine brain mitochondria, as isolated) or only with Type B sites (after treatment with Glc-6-P) prior to detergent treatment, we suggest that the $\mathrm{Mg}^{++}$-dependent interaction of hexokinase with a hydrophobic mitochondrial component (or components) occurs after dissolution of the mitochondria with Triton X-114; interactions within the homogeneous milieu would not be dependent on the status of hexokinase prior to dissolution.

The sensitivity of partitioning behavior to phospholipase treatment implies that one or more phospholipids are involved in conferring hydrophobic character on hexokinase, and the correlation between proportion of Type B sites and partitioning behavior further implies that the relative amount of the responsible phospholipid(s) is similarly correlated with Type B sites. Gross differences in phospholipid composition of bovine and rat brain mitochondria were not seen, but it is certainly conceivable that interaction with hexokinase may depend on more subtle factors. Unfortunately, attempts to identify specific phospholipids that partition with the hexokinase into the detergent phase are doomed since all hydrophobic components, associated with hexokinase or otherwise, are found in the hy- 
drophobic phase. Thus, further studies on this question will require an alternative experimental approach.

\section{R E F E R E N C E S}

1. Katzen, H.M. (1967) The multiple forms of mammalian hexokinase and their significance to the action of insulin. Adv. Enzyme Regul. 5, $335-356$.

2. Wilson, J.E. \& Felgner, P.L. (1977) An inverse relation between mitochondrial hexokinase content and phosphoglucomutase activity of rat tissues. Mol. Cell. Biochem. 18, 39-47.

3. Clarke, D.D. \& Sokoloff, L. (1994) Circulation and energy metabolism in the brain; in Basic Neurochemistry (Siegel, G.J., Agranoff, B.W., Albers, R.W. \& Molinoff, P.B., eds.) pp. 645680, Raven Press, New York.

4. Crane, R.K. \& Sols, A. (1953) The associacion of hexokinase with particulate fractions of brain and other tissue homogenates. J. Biol. Chem. 203, 273-292.

5. Johnson, M.K. (1960) The intracellular distribution of glycolytic and other enzymes in rat-brain homogenates and mitochondrial preparations. Biochem. J. 77, 610-618.

6. Schwartz, G.P. \& Basford, R.E. (1967) The isolation and purification of solubilized hexokinase from bovine brain. Biochemistry 6, 1070-1079.

7. Bachelard, H.S. (1967) The subcellular distribution and properties of hexokinase in the guinea-pig cerebral cortex. Biochem. J. 104, 286-292.

8. Redkar, V.D. \& Kenkare, U.W. (1972) Bovine brain mitochondrial hexokinase. Solubilisation, purification, and role of sulfhydryl residues. J. Biol. Chem. 247, 7576-7584.

9. Magnani, M., Serafini, G., Stocchi, V., Bossù, M. \& Dacha, M. (1982) Solubilisation, purification, and properties of rabbit brain hexo- kinase. Arch. Biochem. Biophys. 216, 449454.

10. BeltrandelRio, H. \& Wilson, J.E. (1992) Coordinated regulation of cerebral glycolytic and oxidative metabolism, mediated by mitochondrially bound hexokinase dependent on intramitochondrially generated ATP. Arch. Biochem. Biophys. 296, 667-677.

11. de Cerqueira Cesar, M. \& Wilson, J.E. (1998) Further studies on the coupling of mitochondrially bound hexokinase to intramitochondrially compartmented ATP, generated by oxidative phosphorylation. Arch. Biochem. Biophys. 350, 109-117.

12.Felgner, P.L., Messer, J.L. \& Wilson, J.E. (1979) Purification of a hexokinase-binding protein from the outer mitochondrial membrane. J. Biol. Chem. 254, 4946-4949.

13. Fiek, C., Benz, R., Roos, N. \& Brdiczka, D. (1982) Evidence for identity between the hexokinase-binding protein and the mitochondrial porin in the outer membrane of rat liver mitochondria. Biochim. Biophys. Acta 688, 429-440.

14. Lindén, M. Gellerfors, P. \& Nelson, B.D. (1982) Pore protein and the hexokinase-binding protein from the outer membrane of rat liver mitochondria are identical. FEBS Lett. 141, 189-192.

15. Colombini, M., Blachly-Dyson, E. \& Forte, M. (1996) VDAC, a channel in the outer mitochondrial membrane; in Ion Channels (Narahishi, T., ed.) vol. 4, pp. 169-202, Plenum Press, New York.

16. Kabir, F. \& Wilson, J.E. (1993) Mitochondrial hexokinase in brain of various species: Differences in sensitivity to solubilisation by glucose-6-phosphate. Arch. Biochem. Biophys. 300, 641-650.

17. Felgner, P.L. \& Wilson, J.E. (1977) Effect of neutral salts on the interaction of rat brain hexokinase with the outer mitochondrial 
membrane. Arch. Biochem. Biophys. 182, 282-294.

18. Kabir, F. \& Wilson, J.E. (1994) Mitochondrial hexokinase in brain: Coexistence of forms differing in solubilisation by glucose-6-phosphate on the same mitochondria. Arch. Biochem. Biophys. 310, 410-416.

19. Tolani, A.J. \& Talwar, G.P. (1963) Differential metabolism of various brain regions: Biochemical heterogeneity of mitochondria. Biochem. J. 88, 357-362.

20. Neidle, A., Van den Berg, C.J. \& Grynbaum, A. (1969) The heterogeneity of rat brain mitochondria isolated on continuous sucrose gradients. J. Neurochem. 16, 225-234.

21. Blokhuis, G.G.D. \& Veldstra, H. (1970) Heterogeneity of mitochondria in rat brain. FEBS Lett. 11, 197-199.

22. Wilson, J.E. (1972) The localization of latent brain hexokinase on synaptosomal mitochondria. Arch. Biochem. Biophys. 150, 96-104.

23. De Pinto, V., Ludwig, O., Krause, J., Benz, R. $\&$ Palmieri, F. (1987) Porin pores of mitochondrial outer membranes from high and low eukaryotic cells: Biochemical and biophysical characterization. Biochim. Biophys. Acta 894, 109-119.

24. Wilson, J.E. (1989) Rapid purification of mitochondrial hexokinase from rat brain by a single affinity chromatography step on Affi-Gel Blue. Prep. Biochem. 19, 13-21.

25. Tuttle, J.P. \& Wilson, J.E. (1970) Rat brain hexokinase: A kinetic comparison of soluble and particulate forms. Biochim. Biophys. Acta 212, 185-188.

26. Bordier, C. (1981) Phase separation of integral membrane proteins in Triton X-114 solution. J. Biol. Chem. 256, 1604-1607.

27. Needels, D.L. \& Wilson, J.E. (1983) The identity of hexokinase activities from mitochondrial and cytoplasmic fractions of rat brain homogenates. J. Neurochem. 40, 1134-1143.
28. Bligh, E.G. \& Dyer, W.J. (1959) A rapid method of total lipid extraction and purification. Can. J. Biochem. Physiol. 37, 911-917.

29. Rouser, G. \& Fleischer, S. (1967) Isolation characterisation, and determination of polar lipids of mitochondria. Methods Enzymol. 10, 385-406.

30.Skipski, V.P. \& Barclay, M. (1969) Thin-layer chromatography of lipids. Methods Enzymol. 14, 530-598.

31. Parlo, R.A. \& Coleman, P.S. (1984) Enhanced rate of citrate export from cholesterol-rich hepatoma mitochondria. The truncated Krebs cycle and other metabolic ramifications of mitochondrial membrane cholesterol. J. Biol. Chem. 259, 9997-10003.

32. Singer, S.J. \& Nicolson, G.L. (1972) The fluid mosaic model of the structure of cell membranes. Science 175, 720-731.

33. Ureta, T., Smith, A.D. \& Wilson, J.E. (1986) Hexokinase A from mammalian brain: Comparative peptide mapping and immunological studies with monoclonal antibodies. Arch. Biochem. Biophys. 246, 419-427.

34. Moller, F. \& Wilson, J.E. (1983) The influence of specific phospholipids on the interaction of hexokinase with the outer mitochondrial membrane. J. Neurochem. 41, 1109-1118.

35. Dorbani, L., Jancsik, V., Linden, M., Leterrier, J.F., Nelson, B.D. \& Rendon, A. (1987) Subfractionation of the outer membrane of rat brain mitochondria: Evidence for the existence of a domain containing the porin-hexokinase complex. Arch. Biochem. Biophys. 252, $188-196$.

36. Parry, D.M. \& Pedersen, P.L. (1990) Glucose catabolism in brain. Intracellular localization of hexokinase. J. Biol. Chem. 265, 1059-1066.

37. Nishikawa, M., Nojima, S., Akiyama, T., Sankawa, U. \& Inoue, K. (1984) Interaction of digitonin and its analogs with membrane cholesterol. J. Biochem. (Tokyo) 96, 1231-1239. 
38. Brdiczka, D. (1990) Interaction of mitochondrial porin with cytosolic proteins. Experientia 46, 161-167.

39. Kottke, M., Adam, V., Riesinger, I., Bremm, G., Bosch, W., Brdiczka, D., Sandri, G. \& Panfili, E. (1988) Mitochondrial boundary membrane contact sites in brain: Points of hexokinase and creatine kinase location, and control of $\mathrm{Ca}^{2+}$ transport. Biochim. Biophys. Acta 935, 87-102.

40. Brdiczka, D. (1991) Contact sites between mitochondrial envelope membranes. Structure and function in energy- and protein-transfer. Biochim. Biophys. Acta 1071, 291-312.

41. Craven, P.A., Goldblatt, P.J. \& Basford, R.E. (1969) Brain hexokinase. The preparation of inner and outer mitochondrial membranes. Biochemistry 8, 3525-3532.
42. Karnovsky, M.J., Kleinfeld, A.M., Hoover, R.L. \& Klausner, R.D. (1982) The concept of lipid domains in membranes. J. Cell Biol. 94, $1-6$.

43. Hinderliter, A.K., Huang, J. \& Feigenson, G.W. (1994) Detection of phase separation in fluid phosphatidylserine/phosphatidylcholine mixtures. Biophys. J. 67, 1906-1911.

44. Brown, D.A. \& London, E. (1997) Structure of detergent-resistent membrane domains: Does phase separation occur in biological membranes? Biochem. Biophys. Res. Commun. 240, $1-7$.

45. Boggs, J.M., Wood, D.D., Moscarello, M.A. \& Papahadjopoulos, D. (1977) Phase separation of acidic and neutral phospholipids induced by human myelin basic protein. Biochemistry 16, 2325-2329. 